BULLETIN Bulletin hispanique

HISPANIQUE Université Michel de Montaigne Bordeaux

118-2 | 2016

Varia

\title{
Philippe Rabaté et Hélène Tropé (éds.), Autour de « Don Quichotte » de Miguel de Cervantès
}

Presses de la Sorbonne Nouvelle, Paris, 2015

\section{Sarah Voinier}

\section{OpenEdition}

\section{Journals}

Édition électronique

URL : http://journals.openedition.org/bulletinhispanique/4677

DOI : 10.4000/bulletinhispanique.4677

ISSN : $1775-3821$

\section{Éditeur}

Presses universitaires de Bordeaux

Édition imprimée

Date de publication : 15 décembre 2016

Pagination : 729-732

ISBN : 979-10-300-0125-9

ISSN : 0007-4640

\section{Référence électronique}

Sarah Voinier, « Philippe Rabaté et Hélène Tropé (éds.), Autour de « Don Quichotte » de Miguel de Cervantès », Bulletin hispanique [En ligne], 118-2 | 2016, mis en ligne le 15 décembre 2016, consulté le 23 septembre 2020. URL : http://journals.openedition.org/bulletinhispanique/4677 ; DOI : https:// doi.org/10.4000/bulletinhispanique.4677

Ce document a été généré automatiquement le 23 septembre 2020

Tous droits réservés 


\section{Philippe Rabaté et Hélène Tropé (éds.), Autour de « Don Quichotte » de Miguel de Cervantès}

Presses de la Sorbonne Nouvelle, Paris, 2015

\section{Sarah Voinier}

\section{RÉFÉRENCE}

Philippe Rabaté et Hélène Tropé (éds.), Autour de «Don Quichotte » de Miguel de Cervantès, Paris, Presses de la Sorbonne Nouvelle, coll. Travaux du Centre de Recherche sur l'Espagne des XVI et ${ }^{\mathrm{e}}$ XVII ${ }^{\mathrm{e}}$ siècles, 2015, $202 \mathrm{p}$.

1 Les Presses de la Sorbonne Nouvelle publient les actes du colloque international que les éditeurs Philippe Rabaté et Hélène Tropé ont organisé à Paris, en janvier 2015, dans le cadre institutionnel des Centres de recherches du CRES de l'Université de Paris 3Sorbonne Nouvelle et du GREAC de l'Université de Paris-Ouest Nanterre La Défense.

2 La rencontre scientifique participa des nombreuses et heureuses manifestations qu'occasionna la célébration du quatrième centenaire de la parution de la Seconde Partie de Don Quichotte de Miguel de Cervantès. Les dix-sept contributions, écrites en français et en espagnol, dans un équilibre linguistique remarquable, s'adressent à la fois aux chercheurs cervantistes et aux étudiants, en particulier aux hispanistes qui se préparent aux concours du capes et de l'agrégation externes d'espagnol des sessions 2015 et 2016. En se saisissant de problématiques au cœur des recherches internationales, ce livre s'inscrit dans le prolongement des recherches de Jean Canavaggio et d'Augustin Redondo, véritables inspirateurs de cette initiative, dont les réflexions inaugurent la première et la dernière partie de l'ensemble des articles.

D'emblée, il faut saluer l'effort de cohérence et de clarté dans la présentation extrêmement précise des travaux. En replaçant l'œuvre dans le contexte des relations hispano-françaises du début $\mathrm{du}$ XVII ${ }^{\mathrm{e}}$ siècle, le lecteur comprend clairement la 
réception immédiatement favorable du Don Quichotte en France. À la faveur de la domination politique de la Monarchie hispanique au XVI ${ }^{\mathrm{e}}$ siècle, et de l'hispanophilie culturelle au siècle suivant, le roman inspire les auteurs français, non seulement par ses «nouvelles intercalées ", mais également par la force inventive de son personnage, d'où les multiples adaptations, traductions, et réinventions à partir du modèle cervantin jusqu'au XX $X^{e}$ siècle.

4 L'ouvrage a pour ambition de rendre compte de l'état de la recherche internationale sur le roman dont la lecture se renouvelle au fil des siècles. Le volume aspire à fournir " un panorama très suggestif tant des problématiques qui traversent l'œuvre, telles que les questions de la justice et de la violence, du rire, que des aspects plus spécifiquement littéraires : les genres littéraires qu'elle parodie et subvertit jusqu'à donner naissance à une nouvelle poétique, ainsi que la question inhérente à toute littérature qui est celle des systèmes de représentation et, concernant ce roman, le perspectivisme qui fonde une nouvelle vision du monde, désormais non unique ni univoque mais au contraire plurielle et variant selon le sujet regardant ». Le pari est réussi.

5 Suivant une articulation en trois volets très finement organisée et équilibrée, l'ouvrage s'ouvre sur "L'intertextualité », en abordant la question des sources et des différents genres qui influencèrent Cervantès dans l'écriture de Don Quichotte. Par son étude, Augustin Redondo redonne toute sa légitimité narrative à l'épisode taurin et à la porcine aventure où les malheurs du chevalier errant s'inscrivent dans le principe de la métamorphose associé au personnage d'Hercule et à ses travaux. En dévoilant les références mythiques et folkloriques qui sous-tendent le texte, l'étude révèle la signification profonde de ces adversités dans l'itinéraire de dégradation que suit le héros déchu. Dans une enquête minutieuse, Hélène Tropé et Luc Torres suivent la piste des emprunts et des écarts dans la forme et le fond entre Palmerin d'Angleterre et Don Quichotte. L'analyse permet ainsi de mieux saisir les variations autour de la filiation littéraire existante entre le roman cervantin et les grands romans de chevalerie tels Amadis de Gaule, et Tirant lo Blanch. Isabel Lozano-Renieblas invite, pour sa part, à analyser le procédé d'inversion générique auquel s'adonne Cervantès dans les noces de Camacho. En partant de la tradition littéraire de la pastorale orale et, en particulier, de l'épithalame, l'auteur montre comment l'imitation burlesque relie culture savante et culture populaire. La réflexion de Juan Diego Vila se fonde également sur les genres littéraires à partir des deux derniers chapitres de l'œuvre. Que ce soit par le modèle homérique pour Sancho ou le modèle pastoral pour Don Quichotte, les personnages tentent de se soustraire à leur triste destin qui les éloigne du métier des armes. Le dénouement et ses tentatives d'échappées génériques soulignent ainsi l'impuissance de leur expérience vitale en renforçant les signes de la mélancolie. Philippe Rabaté ouvre des pistes de réflexion essentielles pour comprendre les différents régimes temporels dans une étude comparative du Guzmán de Alfarache et de Don Quichotte. En partant d'une ébauche de "stratégie de différenciation" de la part de Cervantès dans le maniement des références temporelles, l'analyse prend en considération la genèse de chacun des personnages, et le temps de leurs expériences, mettant ainsi en lumière la part d'anachronisme et d'actualité dans chacune des créations romanesques. La première partie de l'ouvrage se clôt sur une seconde approche comparative puisque David Álvarez Roblin s'interroge sur la riposte que Cervantès offre à son continuateur Avellaneda. En montrant comment l'auteur original tantôt dénigre explicitement son concurrent, tantôt établit un dialogue entre les fictions, tantôt s'en inspire comme une 
«source littéraire » pleine et entière, l'étude renouvelle la lecture traditionnelle et abusive de l'animosité entre les deux auteurs.

6 La deuxième partie du volume, consacrée à l' " élaboration d'une nouvelle poétique ", s'ouvre sur la contribution de Michel Moner, éminent cervantiste de l'hispanisme français auquel l'organisation interne du volume rend également hommage. À travers l'étude des paradoxes du «paratexte verbal » qui encadre le récit du Captif Ruy Pérez de Viedma, l'auteur démonte l'apparente cohérence d'un récit présenté comme véridique et invite au déchiffrement des contradictions internes au texte. Marina Mestra Zaragoza reprend, quant à elle, une problématique déjà bien étudiée, celle du rire et de son rôle dans le Don Quichotte, et se propose d'y sonder la dimension cathartique attribuée par López Pinciano à la fiction. Par le rire, Cervantès invite tout lecteur à dépasser le divertissement pour accéder à la réflexion intime sur sa propre condition: la catharsis comique sert la finalité morale du roman. Isabelle Rouane Soupault se penche sur la gestation romanesque à travers une analyse très suggestive $\mathrm{du}$ motif de l'aventure en chambre, récurrente sous des formes diverses tout au long $\mathrm{du}$ récit. Par des jeux d'inclusion et de réclusion, Cervantès s'adonne à une poétique de l'enveloppement convoquant, par le biais de la métaphore, le «creuset du roman en train de s'écrire", l'espace où le personnage prend forme. Avec une approche novatrice, Susan Byrne met en avant la connaissance de Cervantès de la pratique juridique de son temps. En partant de l'étude lexicale des termes relevant de la combinaison des lettres et des lois, l'auteur montre comment dans la première partie contrairement à la deuxième-, Don Quichotte se porte garant de la justice en redressant le supposé tort royal, notamment lors de l'épisode des galériens. Dans un monde soumis à la force abusive du pouvoir juridictionnel, le chevalier oppose son credo et son action humanistes en libérant les plus faibles du joug des plus forts. Dans le même fil, Bénédicte Torres s'interroge sur les enjeux de la violence omniprésente dans l'œuvre, dont elle affirme qu'elle est, sous ses différentes manifestations, le signe de la fonction sacrificielle de Don Quichotte en butte à un monde chaotique. Le parallèle entre personnage et auteur ne se limite pas aux violences subies mais s'étend à cette volonté commune de défendre, par la violence, un idéal de justice et de liberté. Cette partie du volume se ferme sur une étude de José Montero Reguera centrée sur la revendication du statut de poète par Cervantès. En réponse à des circonstances biographiques et littéraires hostiles, celui-ci complète en l'achevant, dans la deuxième partie du Don Quichotte, un discours amorcé plus tôt dans La Gitanilla et dans le Viaje del Parnaso. L'analyse replace avec clarté la genèse de ce discours et en donne, à partir de sa dernière occurrence, les tenants et les aboutissants.

7 Au début de la troisième partie de l'ouvrage intitulée à « Dire, voir et recevoir le monde de don Quichotte ", Jean Canavaggio s'interroge sur la réception de l'œuvre cervantine à partir de l'épisode, déjà présent chez d'autres contributeurs, de la libération des galériens. L'historique de son interprétation posthume révèle que la dimension comique tend à disparaître avec sa lecture romantique au $\mathrm{XIX}^{\mathrm{e}}$ siècle qui éloigne le lecteur du propos initial établi dans le Prologue de 1605. Il convient aujourd'hui de prendre en compte la mythologie fondée sur la variété des figurations signifiantes que provoquent l'invention narrative de Cervantès et les multiples réceptions qu'elles occasionnèrent pour revenir à l'objet initial de la burla. C'est sur l'intention, avec sa dimension double, que se penche également José Manuel Martín Morán. En partant de l'exemple des dialogues, dont les répercussions affectent non seulement la construction diégétique, mais aussi le conflit dans la vision du monde et la caractérisation des 
personnages. L'analyse de plusieurs exemples d'emploi de mots «bivocaux » sonde le fonctionnement de la parodie et de ses procédés comiques et donne à voir au lecteur attentif la complexité du monde représenté. Les deux contributions suivantes s'attachent à l'iconographie tant dans le texte du Don Quichotte que dans ses illustrations externes. Mercedes Alcalá Galán rappelle en effet l'importance de la relation entre le texte et l'image au XVI ${ }^{\mathrm{e}}$ siècle dont Cervantès était parfaitement conscient tel que le prouvent son expérimentation constante de l'ekphrasis et la reconnaissance de ses limites. L'importance du visuel dans l'imaginaire fictionnel favorise par ailleurs la création d'illustrations autonomes des personnages, présentes encore aujourd'hui dans l'esprit de chacun. José Manuel Lucía Megías s'intéresse, quant à lui, plus spécifiquement aux représentations iconographiques qui attestent, dès 1605, de la prégnance du substrat de l'imaginaire chevaleresque sur lequel Don Quichotte vient fixer sa singularité, notamment comique. Si en Espagne, son succès déclina au XVII ${ }^{\mathrm{e}}$ siècle, sa réception simultanément très favorable en Europe permit sa grande diffusion, et avec elle, un faisceau d'images, toutes éloquentes de l'interprétation étrangère de la folie du "Chevalier à la Triste Figure ». Dans une même démarche diachronique, Jesús Pérez-Magallón élargit la réflexion sur la réception espagnole et européenne du Don Quichotte jusqu'au XVIII ${ }^{\mathrm{e}}$ siècle, en partant du constat de l'indifférence des novatores quant à la création de leur contemporain. Il fallut attendre la fin du XVII e siècle pour que les auteurs espagnols lui reconnussent une valeur incontestable alors qu'en France et en Angleterre, le roman cervantin fut immédiatement lu et interprété à l'aune du déclin de l'Espagne.

8 À la lecture des différentes analyses, on peut regretter à plusieurs reprises que le format limité des textes, lié aux exigences éditoriales, ne permette pas aux auteurs de déployer plus avant leurs réflexions fondées sur des problématiques ambitieuses. Néanmoins, cette contrainte n'entrave en rien la portée scientifique de l'ensemble dont la richesse et la pertinence des analyses, nourries des meilleures études sur le chefd'œuvre de Miguel de Cervantès et confrontées aux recherches les plus récentes, en offre une lecture renouvelée. De ce point de vue, l'initiative d'Hélène Tropé et de Philippe Rabaté s'avère féconde pour servir non seulement de référence immédiate pour une meilleure compréhension du Don Quichotte, mais également de référence durable à la recherche cervantiste. L'amplitude des angles d'approche de l'ensemble des articles, dans des perspectives tantôt parallèles tantôt croisées, confère une puissante envergure thématique à l'ouvrage où chacun puisera matière à concevoir l'inventivité du Don Quichotte.

\section{AUTEURS}

\section{SARAH VOINIER}

Université d'Artois 